
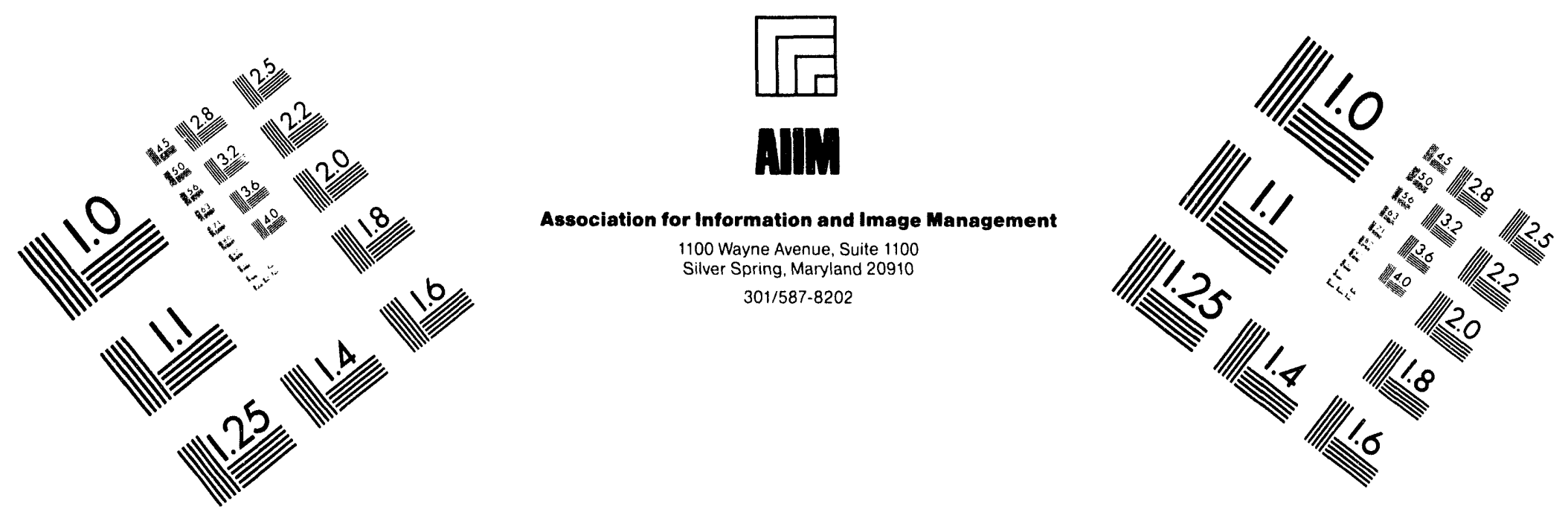

\title{
Centimeter
}

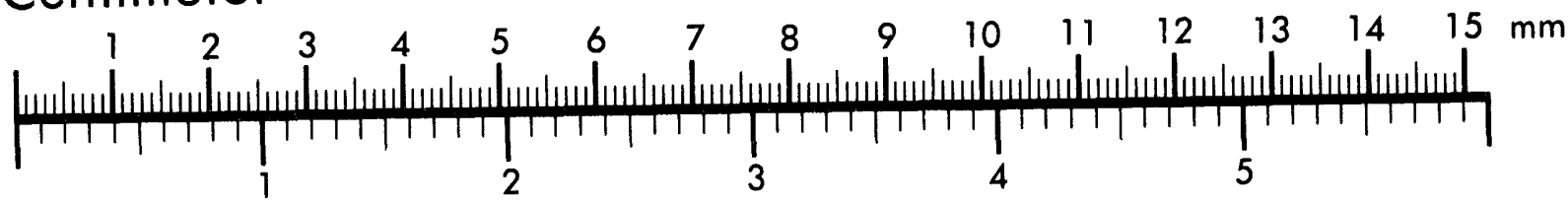
Inches
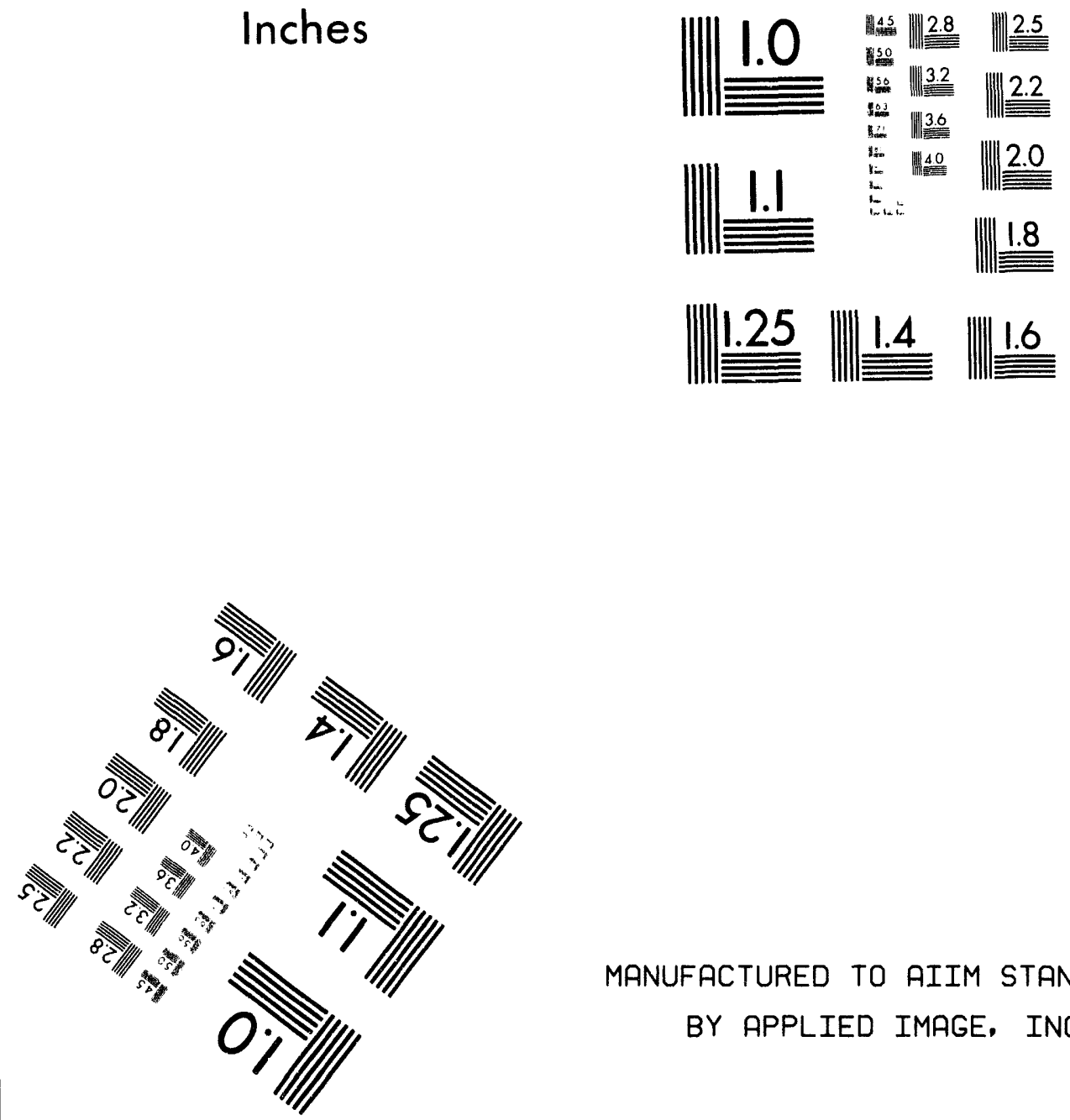

MANUFACTURED TO AIIM STANDARDS

BY APPLIED IMAGE, INC.





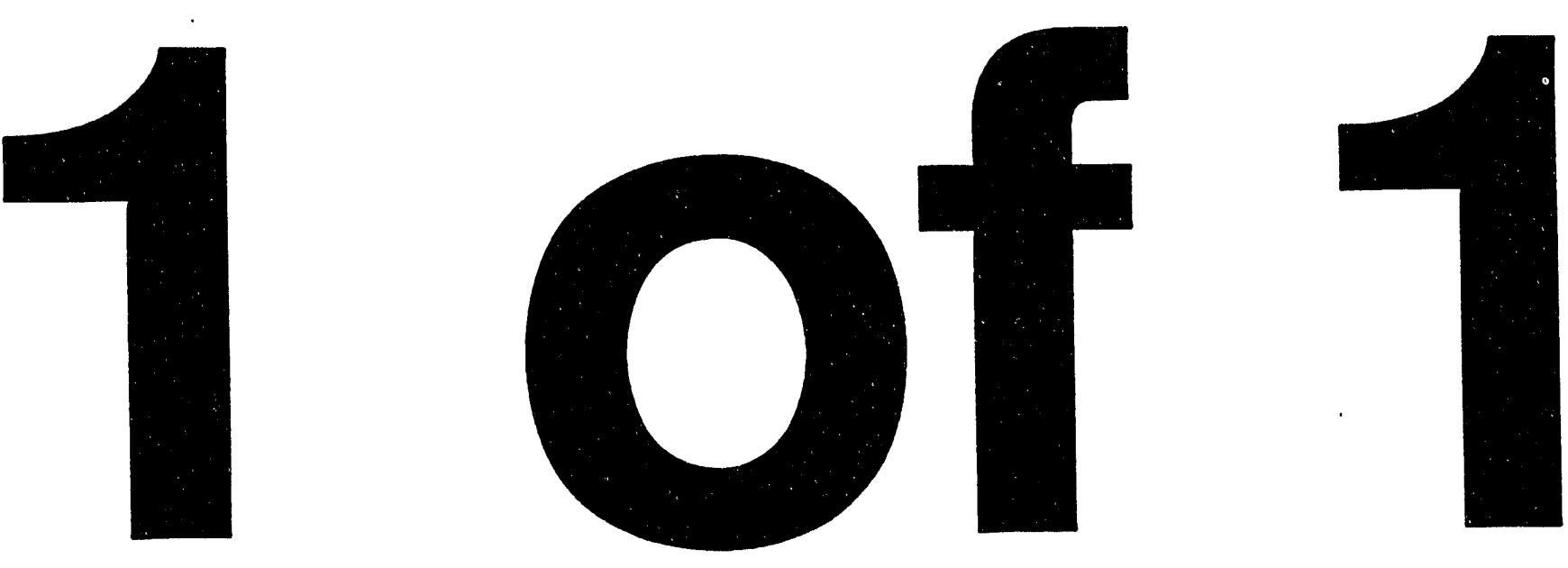


\section{KAON CORRELATION SYSTEMATICS IN E859}

\author{
Ole E. Vossnack \\ Columbia University, New York, NY 10027 and \\ Nevis Laboratories, Irvington, NY 10593, USA \\ for the E859 Experiment (E802 Collaboration) \\ ANL-BNL-UCBerkeley-UCRiverside-Columbia-Hiroshima-INS- \\ Kyushu-LLNL-MIT-NYU-Tokyo
}

PEIVED

MAY 23 POH

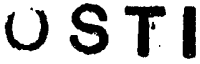

\section{ABSTRACT}

Bose-Einstein correlations between $\mathrm{K}^{+}$'s and between $\pi^{+}$'s produced in collisions of 14.6 A.GeV/c ${ }^{28} \mathrm{Si}$ ions and ${ }^{197} \mathrm{Au}$ nuclei have been measured using the E859 spectrometer. Parameters obtained from fits to correlation functions in relative momentum describe a $\mathrm{K}^{+}$ source that is slightly smaller than the $\pi^{+}$source and comparable in size to the ${ }^{28} \mathrm{Si}$ projectile.

Correlation functions were also formed for $\pi \mathrm{K}^{+}$and $\mathrm{pK}^{+}$pairs extracted from the data set. Bose-Einstein correlations are expected to be absent here. The observed correlations can be understood as being the result primarily of the two-particle Coulomb interaction. For the $\mathrm{pK}^{+}$ pairs, an adequate description is obtained only when taking into account the finite sizes of the $\mathrm{p}$ and $\mathrm{K}^{+}$sources.

The space-time coordinate distributions of pions and kaons produced by the event generator ARC for the $14.6 \mathrm{~A} \cdot \mathrm{GeV} / \mathrm{c}^{28} \mathrm{Si}$ on ${ }^{197} \mathrm{Au}$ collision yield source sizes that are larger than those - observed in the experiment. Much closer agreement is seen when viewing the distributions through a simulated version of the detector.

\section{Introduction}

The symmetrization requirement on the wave function of a multi-particle state causes observable correlations between the particles, which contain information on the space-time geometry of the region in which they were produced ("source"). ${ }^{1}$ The E802 Collaboration has previously reported a measurement of Bose-Einstein correlations between identical pions, both positive and negative, produced in collisions of $14.6 \mathrm{~A} \cdot \mathrm{GeV} / \mathrm{c}^{28} \mathrm{Si}$ projectiles on ${ }^{197} \mathrm{Au}$ and ${ }^{27} \mathrm{Al}$ targets ${ }^{2}$ at the AGS in Brookhaven. All results were consistent with a spherical source that is comparable in size to the ${ }^{28} \mathrm{Si}$ projectile.

As part of Experiment E859, the upgrade of E802, correlations between $\mathrm{K}^{+}$'s have now also been measured, motivated by the belief that the mechanism of their production is different from that of pions and that their re-interaction cross section in nuclear matter is lower, all of which should be reflected in the measured two-particle correlations. ${ }^{3}$ For purposes of comparison, $\pi^{+}$pairs and $\mathrm{K}^{+}$pairs were collected under the same conditions from similar rapidity ranges.

To study the significance of the two-particle Coulomb interaction, correlation functions were formed for $\pi^{+} \mathrm{K}^{+}$and $p \mathrm{~K}^{+}$pairs, where this interaction is expected to be the primary source of correlation. 


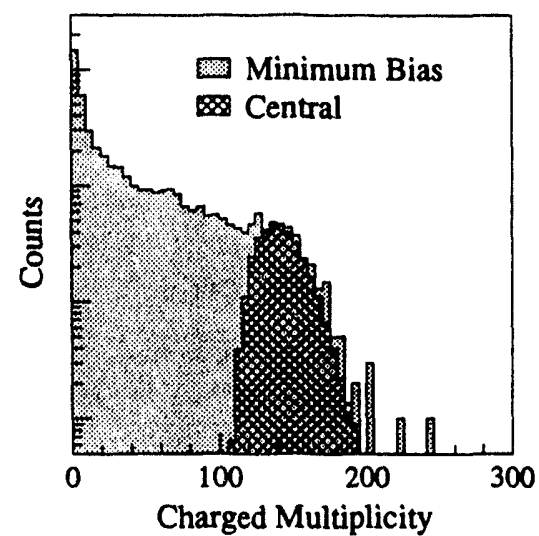

(a)

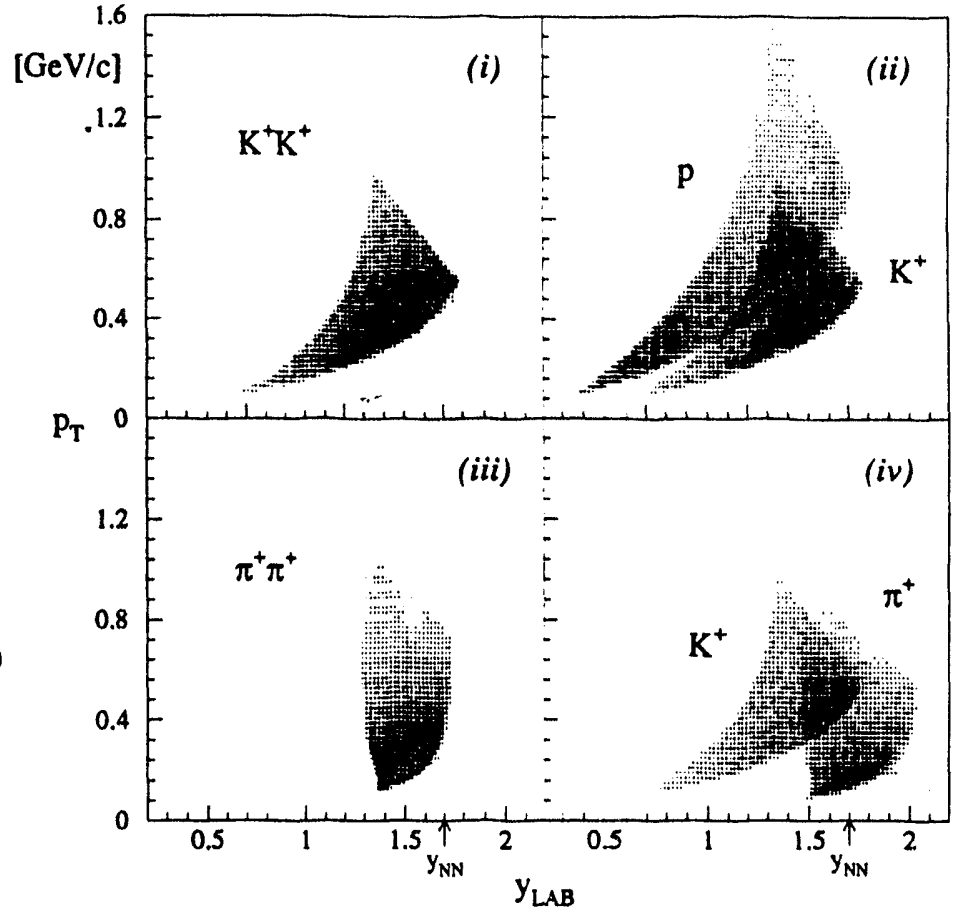

(b)

Fig. 1: a) Charged multiplicity distributions measured by the TMA, for events that satisfied a minimum bias trigger (dotted) and events from the KK data set (hatched). b) Transverse momentum ( $p_{T}$ ) versus rapidity (y) of fully reconstructed pair partners, as measured in the laboratory frame. The $\mathrm{K}^{+} \mathrm{K}^{+}(i), p \mathrm{~K}^{+}(i i)$ and $\pi^{+} \mathrm{K}^{+}(i v)$ pairs are from events collected with the KK trigger with the E859 spectrometer at $14^{0}$ and the $\pi^{+} \pi^{+}\left(\right.$iii) pairs at $19^{0}$.

Event generators can be a useful aid in interpreting the results of a correlation measurement. For example, by explicitly providing the space-time coordinates of produced particles, they allow a direct inspection of the source geometry and, from that, a calibration of the distortion in size and shape of the source resulting from the detection process used in a correlation measurement.

\section{The Experiment}

Using a beam of $\sim 1-2 \times 10^{6} 14.6 \mathrm{~A} \cdot \mathrm{GeV} / \mathrm{c}^{28} \mathrm{Si}$ ions per $\sim 1 \mathrm{~s}$ spill extracted from the AGS as projectiles and a ${ }^{197} \mathrm{Au}$ target with a thickness of $1 \%$ of a ${ }^{28} \mathrm{Si}$ interaction length, events were collected in which the collision produced at least two kaon-like (KK events) or two pion-like tracks $\left(\pi^{+} \pi^{+}\right.$events) in the E859 spectrometer. ${ }^{4}$ The spectrometer consists of a dipole magnet and a total of 46 multiwire planes, placed between the target and a segmented scintillator wall located at $\sim 6 \mathrm{~m}$, with a gas Cerenkov detector directly behind it. The magnet was used at a nominal field setting of $0.4 T$. The geometric acceptance is about $25 \mathrm{msr}$, in the form of a wedge spanning $\sim 14^{\circ}$ horizontally $\left(\theta_{x z}\right)$ and $\sim 7^{\circ}$ vertically $\left(\theta_{y}\right)$. The KK events were collected at the $14^{\circ}$ setting, for which tracks that make angles of $14^{\circ}-28^{\circ}$ with the beam direction enter the aperture. For $\pi^{+} \pi^{+}$events the $19^{\circ}$ setting $\left(19^{\circ}-33^{\circ}\right)$ setting was used in order to end up with pions and kaons of similar rapidity. 
A trigger was implemented that consisted of two consecutive levels. The first level required the coincidence of hits in several detectors, and a sufficient number of charged particles in a detector surrounding use target (TMA) in order to guarantee central collisions (Fig. 1a). The second level selected events based on the information obtained from on-line track reconstruction, momentum measurement and particle identification (PID), which was done using two dedicated multiwire proportional chambers, a segmented scintillator wall and Lecroy ECL logic modules. To select $\mathrm{KK}\left(\pi^{+} \pi^{+}\right)$events, the trigger was programmed to demand the presence of at least 2 tracks that it would identify as kaons ( $\pi^{+}$'s). The resolution and speed of the on-line tracking and PID were such that roughly 1 out of every $14(11)$ events would satisfy the $\mathrm{K}^{+} \mathrm{K}^{+}\left(\pi^{+} \pi^{+}\right)$trigger. Of the fully triggered events, about $0.4 \%(0.8 \%)$ actually yielded pairs. Efficiency and bias studies showed that about $1 \%$ of such "good" events would not satisfy the trigger, but that such "missed" events are not biased in relative momentum. At the available beam intensity, the "rejection power" of the trigger was high enough to allow combinations of rare-event triggers (e.g. $K K \oplus \bar{p})$ within the same run without lowering the collection rate.

\section{Data Set}

During a period in 1991, $5.2 M$ events were collected with a "KK or one anti-proton" trigger ("KK data set") and $\sim 0.9 M$ with a $2 \pi^{+}$trigger (" $\pi \pi$ data set"). After offline track reconstruction and particle identification, with pions and kaons sufficiently separated in inverse velocity $1 / \beta(3 \sigma)$ up to a momentum of $2.2 \mathrm{GeV} / \mathrm{c}$, they yielded $16,444 \mathrm{~K}^{+} \mathrm{K}^{+}$ pairs in 15,719 events and $86,125 \pi^{+} \pi^{+}$pairs in 71,778 events. For studying the two-particle correlations caused by the Coulomb interaction, samples of $25,965 \pi^{+} \mathrm{K}^{+}$and $45,941 \mathrm{pK}^{+}$ pairs were extracted from the KK data set. The results presented here are based on the above pairs. In a second running period, in 1992, additional KK events were collected, the analysis of which is still in progress.

The TMA multiplicity distributions for events from the KK and $\pi \pi$ data sets with at least two fully identified $\mathrm{K}^{+}$or $\pi^{+}$constitute the uppermost $15 \%$ of the distribution for events that satisfy a minimum bias trigger (Fig. 1a), reflecting the centrality cut made by the hardware trigger. ${ }^{5}$ The transverse momentum $\left(p_{T}\right)$ and rapidity $(y)$ distributions of the particles that the various pairs were formed with are shown in Fig. 1b. Note the overlap in coverage between the distributions. for the $\mathrm{K}^{+} \mathrm{K}^{+}$and $\pi^{+} \pi^{+}$pairs. They both peak slightly below the nucleon-nucleon center-of-mass $(\mathrm{CM})$ rapidity $\left(y_{N N}\right)$ of 1.7 .

\section{Correlation Analysis}

For the pairs in the above data set, binned relative momentum distributions were made for particles from the same event ("actual" distribution $A$ ) and particles from different events ("background" distribution $B$ ). Correlation functions were then formed by taking the binby-bin ratio of $A$ and $B$.

To compensate for the effect of the Coulomb interaction in the $\mathrm{K}^{+} \mathrm{K}^{+}$and $\pi^{+} \pi^{+}$pairs, $\mathrm{B}$ was multiplied by the (pointlike) Gamow distribution. For the $\mathrm{pK}^{+}$and $\pi^{+} \mathrm{K}^{+}$pairs, the significance of smearing this correction by the detector resolution and of modifying it for non-pointlike interaction was studied. 

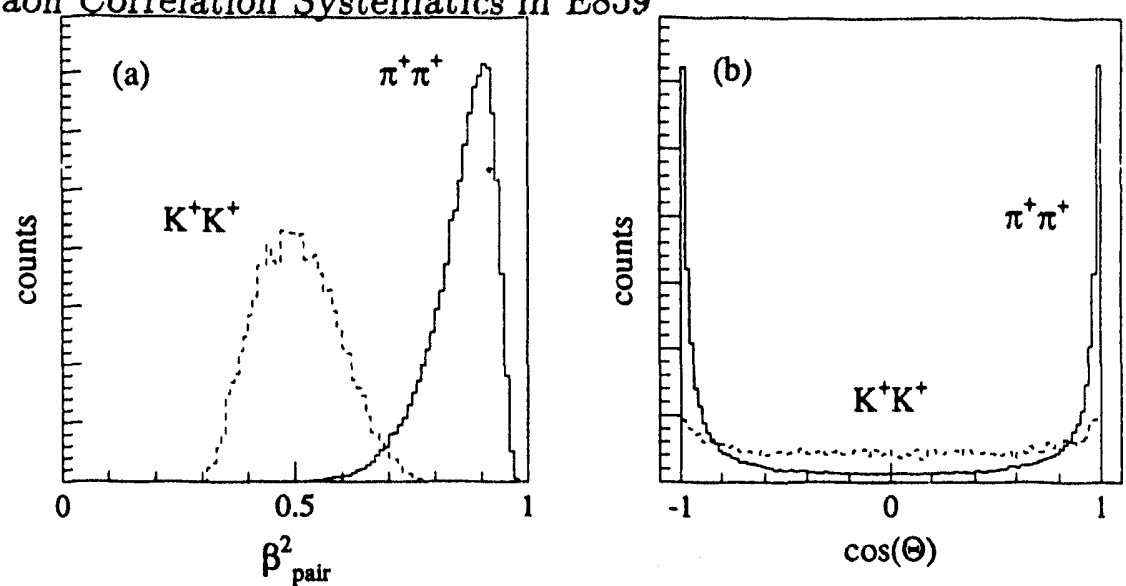

Fig. 2: Pair kinematics in the nucleon-nucleon CM frame for $\pi^{+} \pi^{+}$(solid lines) and $\mathrm{K}^{+} \mathrm{K}^{+}$(dashed lines) pairs: a) histogram of the square of the pair velocity $\left(\beta_{\text {pair }}\right)$; b) histogram of the cosine of the opening angle, $\theta$, between the pair velocity $\left(\vec{\beta}_{\text {pair }}\right)$ and relative momentum $(\vec{q})$ vectors.

The inefficiency (both online and offline) of finding two tracks at a low relative momentum affects $\mathrm{A}$ but not $\mathrm{B}$. To correct for this, correlation functions were made for pairs of particles that were generated independent of each other by Monte Carlo and then passed together through a GEANT simulation of the spectrometer and analyzed as experimental data. The correction then consisted of taking the ratio of the correlation functions from experiment and Monte Carlo.

\section{Results for $\mathrm{K}^{+} \mathrm{K}^{+}$and $\pi^{+} \pi^{+}$pairs}

For a source distribution $\rho(\overrightarrow{x, t})=\exp \left(-|\vec{x}|^{2} / 2 R^{2}-t^{2} / 2 \tau^{2}\right)$ of position vectors, $\vec{x}$, and times, $t$, the correlation function $C_{2}\left(q, q_{0}\right)$ is expected to be given by $1+\exp \left(-q^{2} R^{2}-q_{0}^{2} \tau^{2}\right)$, where $q$ and $q_{0}$ are the spatial and temporal components of the relative momentum in the source rest frame. For lack of sufficient pairs, the correlation functions were analyzed as a function of just one variable, the Lorentz-invariant relative four-momentum, $Q \equiv \sqrt{-\left(p_{1}-p_{2}\right)^{2}}$, where $p_{1}$ and $p_{2}$ are the four-momenta of the particles in any frame. At small $Q$, all relative momentum components must be small, which maximizes the observed correlation.

By fitting the form $f(Q)=N\left(1+\lambda \exp \left(-Q^{2} R_{Q}^{2}\right)\right.$ to the ratio $A(Q) / B(Q)$, parameters $N, \lambda$ and $R_{Q}$ were extracted. $N$ is a normalization and $\lambda$ was inserted to account for a suppression of the correlation due to processes such as final state interactions. ${ }^{1}$ The correlation functions $C_{2}(Q)=A(Q) / B(Q) / \mathrm{N}$ are shown in Fig. 3 before corrections (a and d), after the two-particle acceptance correction (b and e) and fully (i.e. also Gamow) corrected (c and f).

With the signs of the spatial and temporal parts of $Q$ being opposite, $f(Q) / N$ is fundamentally different from $C_{2}\left(q, q_{0}\right)$ (only in the pair CM frame are they equal). Parameter $R_{Q}$ therefore does not directly measure the size of the Gaussian source, i.e. $R_{Q} \neq R$. From the general relation $q_{0}=\vec{q} \cdot \vec{\beta}_{\text {pair }} \equiv|\vec{q}| \beta_{\text {pair }} \cos \theta$ (where $\vec{\beta}_{\text {pair }}$ is the velocity of the pair frame relative to the source reference frame), the parameters $R_{Q}$ and $R$ can be related via

$$
\left(\frac{R_{Q}}{R}\right)^{2}=\frac{1+\left(\frac{\tau^{2}}{R^{2}}\right) \beta_{\text {pair }}^{2} \cos ^{2} \theta}{1-\beta_{\text {pair }}^{2} \cos ^{2} \theta} .
$$




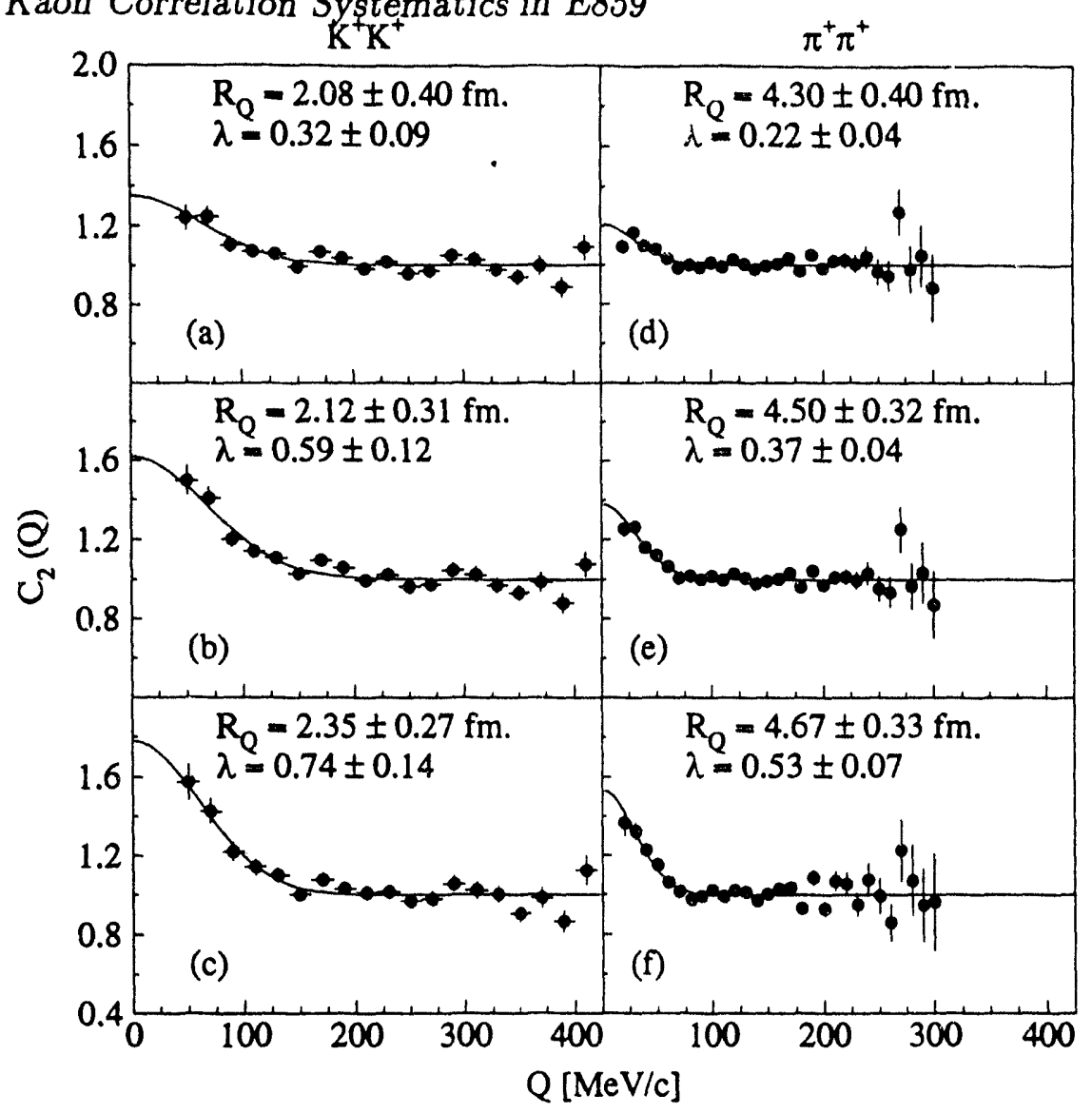

Fig. 3: Correlation functions for $\mathrm{K}^{+} \mathrm{K}^{+}(\mathrm{a}, \mathrm{b}, \mathrm{c})$ and $\pi^{+} \pi^{+}(\mathrm{d}, \mathrm{e}, \mathrm{f})$ pairs, uncorrected(a and d), two-particle acceptance corrected (b and $e$ ) and fully corrected ( $c$ and $f$ ). Note the difference between kaons and pions in the intercept at $Q=0$ and the width of the region of enhancement. The source rest frame was assumed to be at $y_{N N}=1.7$.

The distributions of $\beta_{\text {pair }}^{2}$ and $\cos \theta$ in the nucleon-nucleon CM frame for pairs from the data set are shown in Fig. 2. The average pion pair velocity is higher and more aligned with the relative momentum $\vec{q}$ than the average kaon pair velocity. Assuming $R \approx \tau$ (as was observed by $E 802^{2}$ ), the distributions indicate that $R_{Q} / R$ is different from 1 and larger for pions than for kaons.

Instead of trying to find a correctly weighted average of eq. (1) over the experimental data set, a conversion factor between $R$ and $R_{Q}$ was obtained by a Monte Carlo procedure. Sets of $\pi^{+} \pi^{+}$and $\mathrm{K}^{+} \mathrm{K}^{+}$pairs ("events") were generated by importance sampling from transverse mass $\left(m_{T}=\sqrt{p_{T}^{2}+m^{2}}\right)$ and rapidity distributions similar to those observed in the E802 single particle data and from a Gaussian two-particle distribution in $q$ and $q_{0}$ with $R=2 \mathrm{fm}$ and $\tau=2 \mathrm{fm}$. Only those pairs were kept that would be accepted by the E859 spectrometer. The corresponding $R_{Q}$ 's were then extracted from correlation functions in $Q$ for these pairs, yielding ratios $R / R_{Q}$ of 1.27 for the kaons and 2.06 for the pions. It was verified that the value $R / R_{Q}$ is not very sensitive to the values chosen for $R$ and $\tau$ of the input distribution. (i.e., it is mainly determined by the kinematical relationship between $q, q_{0}$ and $Q$ ).

By assuming that $R=\tau$ and forming a correlation function in the single "variable" $Q_{\tau} \equiv \sqrt{q^{2}+q_{0}^{2}}$, a value for $R$ can be directly obtained by fitting this correlation function 


\begin{tabular}{|c|c|c|c|c|c|}
\hline Fit & System & $F_{\text {kin }}$ & $r_{R M S}[\mathrm{fm}]$ & $\lambda$ & $\chi^{2} /$ dof \\
\hline$Q$ & $\mathrm{~K}^{+} \mathrm{K}^{+}$ & 1.27 & $3.22 \pm 0.37$ & $0.74 \pm 0.14$ & $37.6 / 26$ \\
& $\pi^{+} \pi^{+}$ & 2.06 & $3.93 \pm 0.28$ & $0.53 \pm 0.07$ & $30.7 / 25$ \\
\hline$\sqrt{|\vec{q}|^{2}+q_{0}^{2}}$ & $\mathrm{~K}^{+} \mathrm{K}^{+}$ & 1 & $3.20 \pm 0.36$ & $0.71 \pm 0.13$ & $21.3 / 26$ \\
$R=\tau$ & $\pi^{+} \pi^{+}$ & 1 & $4.78 \pm 0.42$ & $0.56 \pm 0.07$ & $30.3 / 25$ \\
\hline$\vec{q}, q_{0}$ & $\pi^{+} \pi^{+}$ & 1 & $4.42 \pm 0.94$ & $0.53 \pm 0.07$ & $366.0 / 367$ \\
\hline
\end{tabular}

Table 1: Fit parameters for the two data sets. Uncertainties are statistical only. The systematic errors are estimated to be $\pm 0.3 \mathrm{fm}$ in $r_{R M S}$ and \pm 0.1 in $\lambda$.

to the form $f\left(Q_{\tau}\right)=N\left(1+\lambda \exp \left(-Q_{\tau} R^{2}\right)\right.$. The resulting values for $R$ and $\lambda$ are consistent with those obtained from $C_{2}(Q)$.

Sufficient $\pi^{+} \pi^{+}$pairs were available to allow a fit of the correlation function in $q$ and $q_{0}$ with both $R$ and $\tau$ as free parameters. Again, the values of $R$ and $\lambda$ are consistent with those from $C_{2}(Q)$. The results are summarized in Table 1 . The radii are listed as $r_{R M S}=R \sqrt{3}$. The "Monte-Carlo-calibrated" kinematic conversion factor between $R_{Q}$ and $R$ is given in the third column as $F_{k i n}$. See also Ref. 6.

\section{Results for $\pi^{+} \mathrm{K}^{+}$and $\mathrm{pK}^{+}$pairs}

For the $\pi^{+} \mathrm{K}^{+}$and $\mathrm{pK}^{+}$pairs that were extracted from the $\mathrm{KK}$ data set, the transverse momentum and laboratory rapidity distributions are shown in Figs. $1 \mathrm{~b}$.

The correlation here is expected to be mainly due to the Coulomb force between the particles in a pair, with small contributions from the Coulomb interaction with particles from the surrounding matter. ${ }^{7}$ The effective range measured for the $\mathrm{pK}^{+}$(dominantly swave repulsive) strong interaction is $\sim 0.4 \mathrm{fm} .{ }^{8}$ For the $\pi^{+} \mathrm{K}^{+}$system, calculations suggest a similarly small effective range. ${ }^{9}$ For a source of several fermis, the effect of the strong interactions is therefore expected to be small.

Correlation functions were constructed using the relative momentum in the pair $\mathrm{CM}$ frame, $\vec{k}=\left(m_{2} \vec{p}_{1}^{*}-m_{1} \vec{p}_{2}\right) /\left(m_{1}+m_{2}\right)$, since that is the relevant variable for two-particle interaction. "The uncorrected correlation functions, $C_{2}(k)$, are shown in Figs. $4 \mathrm{~b}$ and $\mathrm{c}$.

The Gamow factor, defined as

$$
G(k)=\frac{\left|\Psi_{k}(\vec{r}=0)\right|^{2}}{\left|\Psi_{k}(\vec{r} \rightarrow \infty)\right|^{2}}=\frac{2 \pi \alpha \frac{\mu}{k}}{e^{2 \pi \alpha \frac{\mu}{k}}-1}
$$

where $\Psi_{k}$ is the Schrödinger wave function, $\mu=m_{1} m_{2} /\left(m_{1}+m_{2}\right)$ the relative mass and $\alpha$ the fine structure constant, describes the pair distribution in $k$ when the particles are not

\footnotetext{
* $k$ can be expressed in terms of Lorentz-invariant quantities as $k^{2}=\frac{1}{4}\left[Q^{2}+\frac{\left(m_{1}^{2}-m_{2}^{2}\right)^{2}}{M^{2}}\right]$, where $M$ is the invariant mass of the pair, so that $k$ is just $Q / 2$ for equal-mass particles, and, unlike $Q$, remains real otherwise.
} 


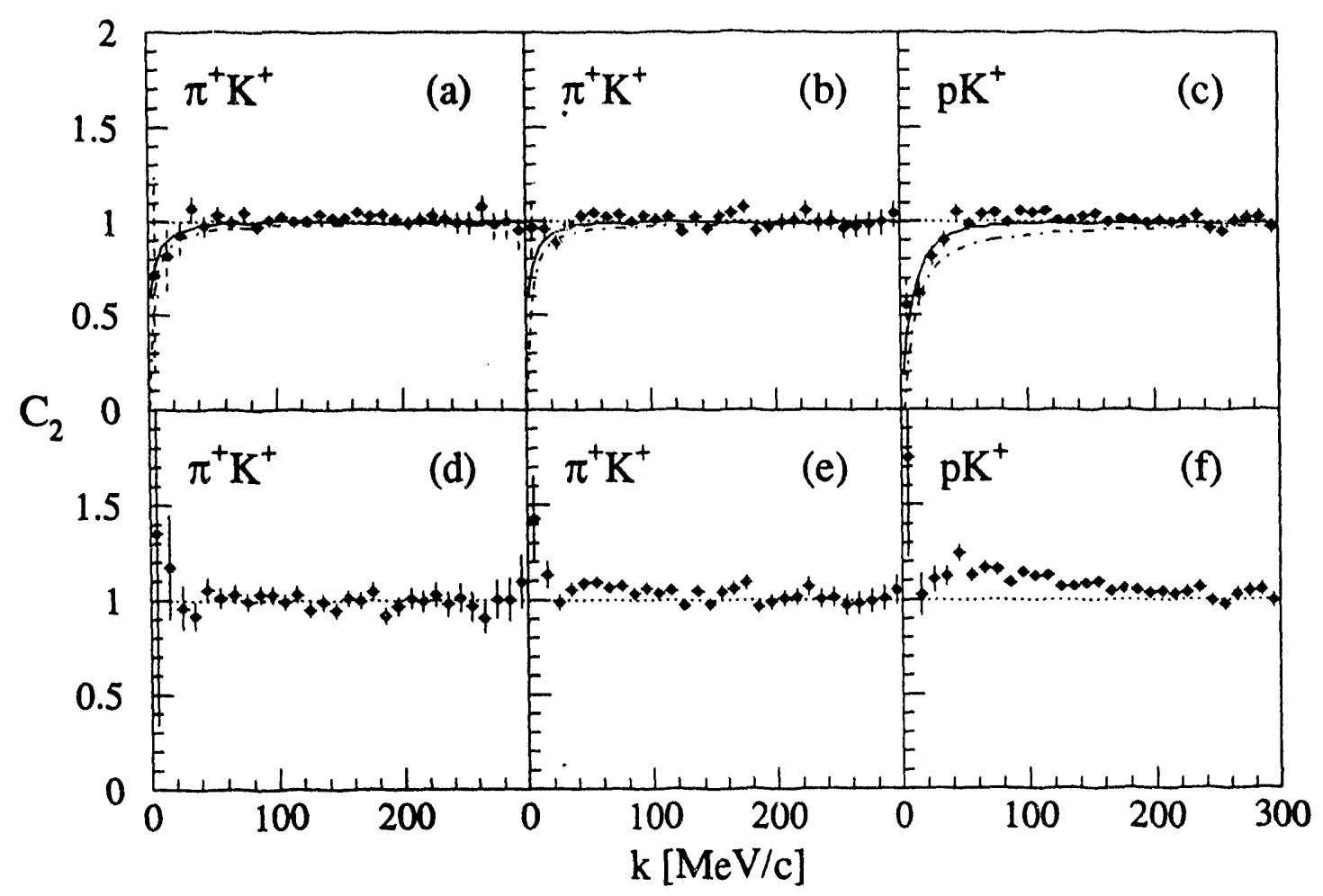

Fig. 4: Correlation functions, $C_{2}(k)$, for $\pi^{+} \mathrm{K}^{+}$and $\mathrm{pK}^{+}$pairs from experiment $(\mathrm{b}, \mathrm{c})$ and Monte Carlo (sampled Gamow) (a); The ratio of (b) and (a) is shown in (d) and the Gamow-corrected versions of (b) and (c) in (e) and (f). The dot-dashed curve is the Gamow factor. The solid curve is a modified Gamow factor for a Gaussian-distributed interparticle separation. The dotted line is at $C_{2}(k)=1$ for reference.

separated in real space, i.e. for a point source. This distribution is shown for the $\pi^{+} \mathrm{K}^{+}$ $\left(\mu=108.81 \mathrm{MeV} / \mathrm{c}^{2}\right)$ and $\mathrm{pK}^{+}\left(\mu=323.46 \mathrm{MeV} / \mathrm{c}^{2}\right)$ pairs in Fig. 4 as dot-dashed curves which lie below the data, especially for the $\mathrm{pK}^{+}$pairs. The Gamow corrected $C_{2}(k)$ are shown in Figs. $4 e$ and $f$.

In the more realistic case of having a distribution, $\rho(\vec{r})$, of spatial separations $\vec{r}$, the Gamow factor is modified into $G^{\prime}(k)$, which is obtained by integration of $\Psi(\vec{r})$ and $\rho(\vec{r}):{ }^{10}$

$$
G^{\prime}(k)=\frac{\int d^{3} r\left|\Psi_{k}(\vec{r})\right|^{2} \rho(\vec{r})}{\left|\Psi_{k}(\vec{r} \rightarrow \infty)\right|^{2}}=G(k)[1+2 \alpha \mu<r>+\cdots]
$$

To approximate $\rho(\vec{r})$ for $\mathrm{pK}^{+}$pairs, coordinates were generated by randomly sampling Gaussian distributions in the spatial coordinates with 3D RMS radii of $3.22 \mathrm{fm}$ for kaons (Table 1) and $4.75 \mathrm{fm}$ for protons ${ }^{13}$ as were observed experimentally. $G^{\prime}(k)$ for these distributions is shown as the solid curve in Fig. 4c and follows the data much better than $G(k)$. Changing the source radii by $\sim 20 \%$ in either direction produced curves that fit less well. The same was done for the $\pi^{+} \mathrm{K}^{+}$pairs using the RMS radii from Table 1, yielding a less significant difference.

Fig. 4a shows $C_{2}(k)$ for $\pi^{+} \mathrm{K}^{+}$pairs that were generated by randomly sampling a Gamow distribution and then passed through a GEANT simulation of the E859 spectrometer and analyzed as experimental data to take into account the resolution smearing. The ratio of 


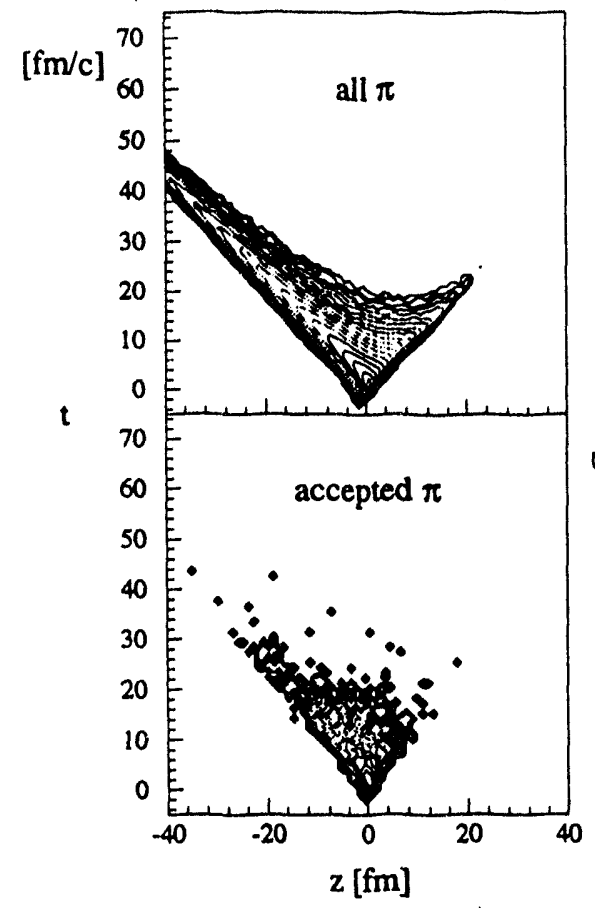

(a)

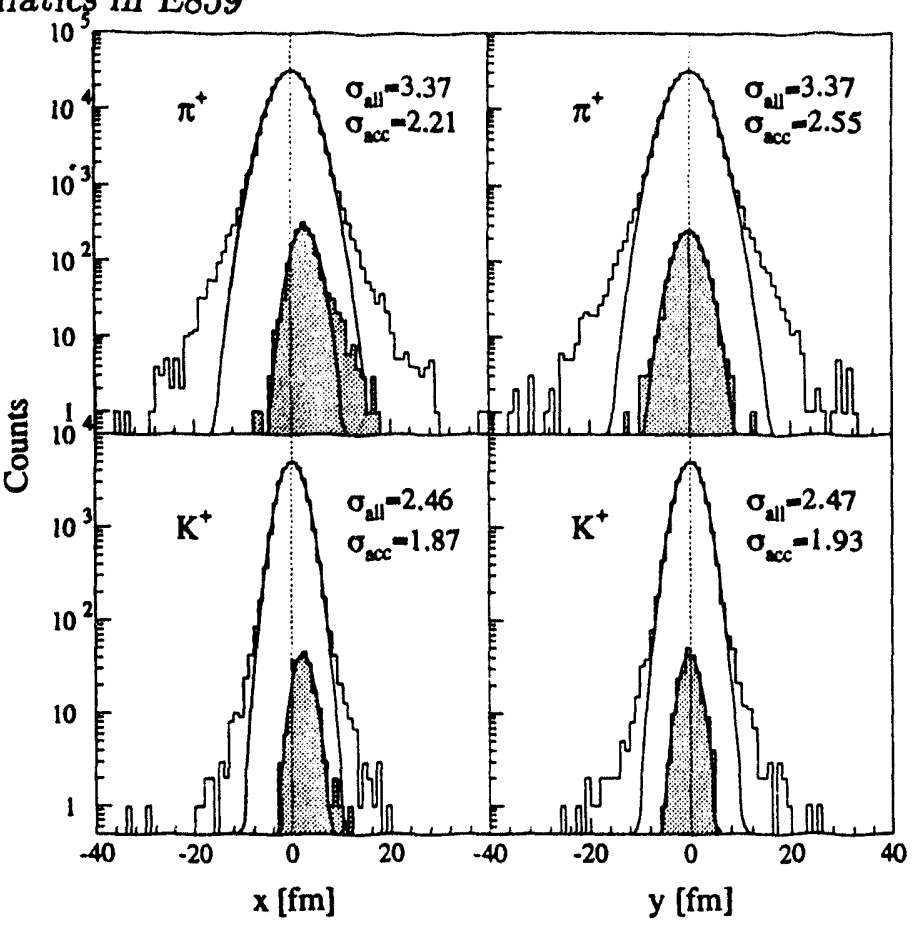

(b)

Fig. 5: a) Logarithmic contour plot of the time and real-space z-coordinates of the points of last interaction for pions produced by ARC in a $14.6 \mathrm{~A} \cdot \mathrm{GeV} / \mathrm{c}^{28} \mathrm{Si}$ on ${ }^{197} \mathrm{Au}$ collision at zero impact parameter, as measured in the nucleon-nucleon CM frame. The upper plot shows all pions produced by ARC, the lower plot those that enter the E859 acceptance. b) Distributions of the $x$ - and $y$-coordinates for produced (clear) and accepted (hatched) $\pi^{+}$and $\mathrm{K}^{+}$. Standard deviations $(\sigma)$ of Gaussian fits are given. The dotted lines are for reference.

the $C_{2}(k)$ in Figs. 4b and a, shown in Fig. $4 \mathrm{~d}$, provides the fully corrected $C_{2}(k)$ for $\pi^{+} \mathrm{K}^{+}$ pairs.

\section{Correlations Measurements and Event Generators}

Even though most event generators do not require wave function symmetrization and therefore will not produce the related correlations, they allow inspection of the source geometry from the real-space coordinates of the produced particles. Assuming that the source geometry is not affected strongly by the absence of "symmetrization" correlations, the size parameters of the real-space distributions can be compared with those obtained from the correlation measurement on the data from the experiment.

A Bose-Einstein correlation measurement determines the distribution of particles at the moment of their last (strong) interactions (source at "freeze-out"). A study was made of the real-space "freeze-out" distributions in a set of $500014.6 \mathrm{~A} \cdot \mathrm{GeV} / \mathrm{c}^{28} \mathrm{Si}$ on ${ }^{197} \mathrm{Au}$ collisions at impact parameter zero as simulated by the event generator ARC. ${ }^{11}$ The $(t, z)$-distributions for pions and the $x$-and $y$-distributions for $\pi^{+}$and $\mathrm{K}^{+}$are shown in Fig. 5. The projectile is coming in along the $z$-axis, the $y$-axis is vertical and the $x$-axis horizontal.

In each case, the effect is shown of requiring that the particles pass a filter that simulates the geometric and momentum acceptance of the E859 spectrometer described in section 2. It is centered on the positive $(x, z)$-plane. Spectrometer settings were chosen to be identical 
to the ones for the E859 KK $\left(14^{\circ}\right)$ and $\pi^{+} \pi^{+}\left(19^{\circ}\right)$ running. Fig. 5a tells us that even though not every produced particle is detected; at least the ones from the interesting region are seen. Note that the $x$ - and $y$-distributions before filtering have non-Gaussian tails. Filtering narrows the $y$-distributions by a factor of $1 / 0.76$ for $\pi^{+'}$ 's and $1 / 0.78$ for $\mathrm{K}^{+}$'s and clips their Gaussian wings. The filtered $x$-distributions are narrower, off-center and asymmetric; here the ratios are $1 / 0.66$ and $1 / 0.76$ respectively. Particles from the "far side" of the source are apparently not detected. Evaluations of $\vec{x} \cdot \vec{p}$ (in the CM frame) and $\vec{x}_{T} \cdot \vec{p}_{T}$ indicate that position and momentum vectors are preferentially aligned. Whether this type of correlation stems from the primary collision dynamics or from a successive absorbtion in the intermediate matter, is not yet clear.

A direct comparison of source sizes with the experimental results is tempting, although the reader is cautioned that the version of ARC used here did not include $\mathrm{K}^{+}$production from $\mathrm{K}^{*}$ decay, which affects the corresponding source size. The average of the standard deviations of Gaussian fits (Fig. 5) to the $x$ - and $y$-distributions times $\sqrt{3}$ is used as an approximation of the 3D RMS radius of the source distribution. Before acceptance filtering, this RMS radius is $4.27 \mathrm{fm}$ for the $\mathrm{K}^{+}$and $5.84 \mathrm{fm}$ for the $\pi^{+}$source. After filtering, the $\mathrm{K}^{+}$ radius becomes $3.29 \mathrm{fm}$ and the $\pi^{+}$radius $4.12 \mathrm{fm}$. The latter values agree very well with the experimental results in Table 1. Results for protons are also consistent, with $6.45 \mathrm{fm}$ for the unfiltered and $4.27 \mathrm{fm}$ for the filtered ARC source versus $4.50 \pm 0.14$, the fully Lorentz corrected value from experimental observation. ${ }^{13} \mathrm{~A}$ similar study using the event generator RQMD, ${ }^{12}$ which handles the collision dynamics differently, is in progress.

\section{Conclusion}

The $\mathrm{K}^{+}$source radius was measured to be somewhat smaller then the $\pi^{+}$source radius. From the analysis of $\pi^{+} \mathrm{K}^{+}$and $\mathrm{pK}^{+}$pairs, it appears that the contribution from Coulomb interaction to the $\pi^{+} \pi^{+}$and $\mathrm{K}^{+} \mathrm{K}^{+}$correlation functions can be reliably separated from the Bose-Einstein part by means of the Gamow correction. Taking into account the smearing due to detector resolution improves this correction. The $\mathrm{pK}^{+}$correlation was seen to be sensitive to the finite size of $\mathrm{p}$ and $\mathrm{K}^{+}$sources. In the (near) future, event generators may prove useful in calibrating the results from a correlation measurement.

\section{Acknowledgements}

Experiment E802 is supported in part by the U.S. Department of Energy contracts and grants with ANL, BNL, UC-Berkeley, UC-Riverside, Columbia, LLNL, and MIT, in part by NASA under contract with UC-Berkeley and by the US-Japan High Energy Physics Collaboration treaty.

\section{References}

1. For recent reviews, see W.A. Zajc, in Hadronic Multiparticle Production, World Scientific Press, P. Carruthers, ed. (1988). D.H. Boal, C.K. Gelbke and B.K. Jennings, Rev. Mod. Phys. 62, 553 (1990).

2. T. Abbott et al., Phys. Rev. Lett. 69, 1030 (1992).

3. Proposal for experiment 859, BNL-P859, 32ff 1989. 
to the ones for the E859 KK $\left(14^{\circ}\right)$ and $\pi^{+} \pi^{+}\left(19^{\circ}\right)$ running. Fig. 5a tells us that even though not every produced particle is detected, at least the ones from the interesting region are seen. Note that the $x$ - and $y$-distributions before filtering have non-Gaussian tails. Filtering narrows the $y$-distributions by a factor of $1 / 0.76$ for $\pi^{+'} \mathrm{~s}$ and $1 / 0.78$ for $\mathrm{K}^{+}$'s aud clips their Gaussian wings. The filtered $x$-distributions are narrower, off-center and asymmetric; here the ratios are $1 / 0.66$ and $1 / 0.76$ respectively. Particles from the "far side" of the source are apparently not detected. Evaluations of $\vec{x} \cdot \vec{p}$ (in the CM frame) and $\vec{x}_{T} \cdot \vec{p}_{T}$ indicate that position and momentum vectors are preferentially aligned. Whether this type of correlation stems from the primary collision dynamics or from a successive absorbtion in the intermediate matter, is not yet clear.

A direct comparison of source sizes with the experimental results is tempting, although the reader is cautioned that the version of $\mathrm{ARC}$ used here did not include $\mathrm{K}^{+}$production from $\mathrm{K}^{*}$ decay, which affects the corresponding source size. The average of the standard deviations of Gaussian fits (Fig. 5) to the $x$ - and $y$-distributions times $\sqrt{3}$ is used as an approximation of the 3D RMS radius of the source distribution. Before acceptance filtering, this RMS radius is $4.27 \mathrm{fm}$ for the $\mathrm{K}^{+}$and $5.84 \mathrm{fm}$ for the $\pi^{+}$source. After filtering, the $\mathrm{K}^{+}$ radius becomes $3.29 \mathrm{fm}$ and the $\pi^{+}$radius $4.12 \mathrm{fm}$. The latter values agree very well with the experimental results in Table 1. Results for protons are also consistent, with $6.45 \mathrm{fm}$ for the unfiltered and $4.27 \mathrm{fm}$ for the filtered ARC source versus $4.50 \pm 0.14$, the fully Lorentz corrected value from experimental observation. ${ }^{13} \mathrm{~A}$ similar study using the event generator $\mathrm{RQMD},{ }^{12}$ which handles the collision dynamics differently, is in progress.

\section{Conclusion}

The $\mathrm{K}^{+}$source radius was measured to be somewhat smaller then the $\pi^{+}$source radius. From the analysis of $\pi^{+} \mathrm{K}^{+}$and $\mathrm{pK}^{+}$pairs, it appears that the contribution from Coulomb interaction to the $\pi^{+} \pi^{+}$and $\mathrm{K}^{+} \mathrm{K}^{+}$correlation functions can be reliably separated from the Bose-Einstein part by means of the Gamow correction. Taking into account the smearing due to detector resolution improves this correction. The $\mathrm{pK}^{+}$correlation was seen to be sensitive to the finite size of $\mathrm{p}$ and $\mathrm{K}^{+}$sources. In the (near) future, event generators may prove useful in calibrating the results from a correlation measurement.

\section{Acknowledgements}

Experiment E802 is supported in part by the U.S. Department of Energy contracts and grants with ANL, BNL, UC-Berkeley, UC-Riverside, Columbia, LLNL, and MIT, in part by NASA under contract with UC-Berkeley and by the US-Japan High Energy Physics Collaboration treaty.

\section{References}

1. For recent reviews, see W.A. Zajc, in Hadronic Multiparticle Production, World Scientific Press, P. Carruthers, ed. (1988). D.H. Boal, C.K. Gelbke and B.K. Jennings, Rev. Mod. Phys. 62, 553 (1990).

2. T. Abbott et al., Phys. Rev. Lett. 69, 1030 (1992).

3. Proposal for experiment 859, BNL-P859, 32ff 1989. 
4. The E859 spectrometer is an upgrade of the E802 spectrometer, described in T. Abbott et al., NIM A290, 41 (1990).

5. This cut was made both by requiring sufficient TMA hits and the presence two koan or pion-like tracks in the spectrometer; see also the contribution of R.A. Soltz.

6. Y. Akiba et al., Phys. Rev. Lett. 70, 1057 (1993).

7. M. Gyulassi and S.K. Kauffmann, Nucl. Phys. A418, 59 (1981).

8. R.A. Burnstein et al., Phys. Rev. D10, 2767 (1974).

9. R.W. Griffith, Phys. Rev. 176, 1705 (1968).

10. W.A. Zajc, PhD thesis and S.Pratt, private communications.

11. Y. Pang, T.J. Schlagel and S.H. Kahana, Phys. Rev. Lett. 68, 2743 (1992).

12. R. Mattiello, H. Sorge, H. Stöcker and W. Greiner, Phys. Rev. Lett. 63, 1459 (1989).

13. Recent Results from E802 and E859, W.A. Zajc, In Proceedings of Quark Matter 91, Nucl. Phys. A544 (1992)237c-251c.

For more recent analysis of the proton data, see the contribution by Stephans to these proceedings.

\section{DISCLAIMER}

This report was prepared as an account of work sponsored by an agency of the United States Government. Neither the United States Government nor any agency thereof, nor any of their employees, makes any warranty, express or implied, or assumes any legal liability or responsibility for the accuracy, completeness, or usefulness of any information, apparatus, product, or process disclosed, or represents that its use would not infringe privately owned rights. Reference herein to any specific commercial product, process, or service by trade name, trademark, manufacturer, or otherwise does not necessarily constitute or imply its endorsement, recommendation, or favoring by the United States Government or any agency thereof. The vie:ws and opinions of authors expressed herein do not necessarily state or reflect those of the United States Government or any agency thereof. 

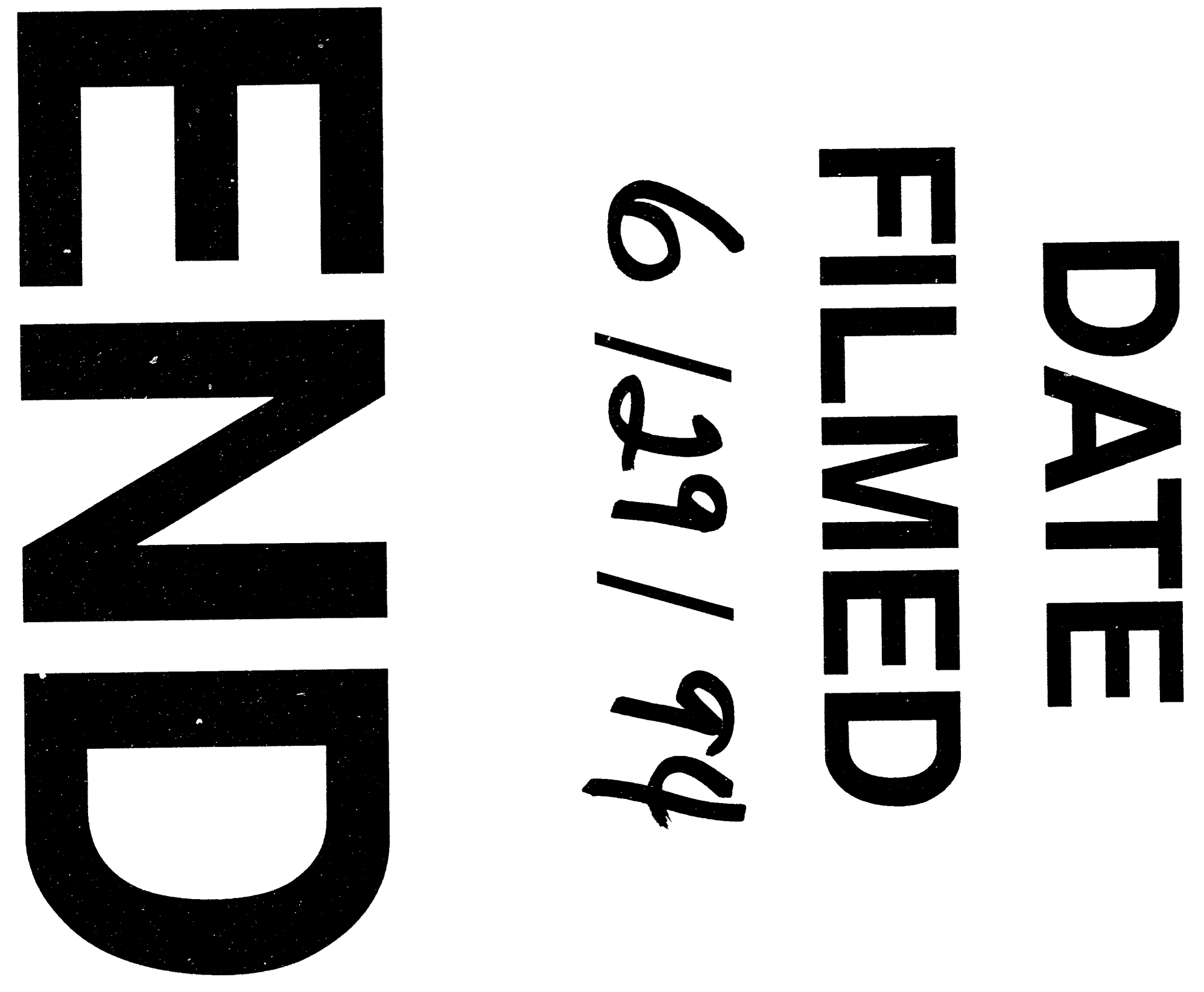
